\title{
Design of Networked Microgrid's Control towards Power Coordination and Voltage - Frequency Regulation
}

\author{
Nadil Amin, K. Hasan
}

\begin{abstract}
One of the state-of-the-arts of smart grid system is the concept of networked microgrid that provides flexible integration and coordination of distributed renewable energy resources in multiple microgrids. However, due to the variable nature of renewable generations, load changes and grid contingencies, the system suffers from significant fluctuations of power, voltage and frequency. This paper seeks to present comprehensive converter control solutions for power sharing coordination and stabilization of voltages and frequency to ensure high power quality and reliability in a networked microgrid system regardless of its grid-connected and islanded modes of operation. The control solutions are provided in $d-q$ axes for both active and reactive powers. The multilevel inverter topology that is designed to interface the distributed energy resources results in better suppression of output harmonics compared to that with a conventional 2-level inverter. The paper also features an interlinking algorithm framework of particle swarm optimization (PSO) to tune the control parameters for the optimized performance of converters. A prototype of grid-connected system of three microgrids is simulated in PSCAD platform to validate the efficacy of the proposed solution.
\end{abstract}

Keywords : Networked microgrid, load sharing, voltage control, decentralized, PSO, frequency control.

\section{INTRODUCTION}

Thegrowing number of microgrids due to the increased penetration of renewable energy sources (RES) has emerged the concept of networked operation of different self-governed microgrids in the modern power systems worldwide. As per the projection of the International Renewable Energy Agency (IRENA) in 2018, the global RES capacity would get doubled of the contemporary $2179 \mathrm{GW}$ generation [1]. Microgrids (MGs) serve as essential electrical infrastructures to integrate these cheap and clean distributed energy resources (DERs) by considerably reducing the harmful alternatives like coal fired and nuclear power plants that got $\mathrm{CO} 2$, SO2 and other polluted emissions [2]. While microgrids, in their grid-mode of operation, ensure cost-effective power quality by exporting and importing to the main grid as singular entities, they also provide uninterrupted power supply to the potential customers under

Revised Manuscript Received on April 21, 2020.

* Correspondence Author

Nadil Amin*, Department of Electrical Engineering, The University of Sydney, Sydney, Australia. E-mail: nami4717@uni.sydney.edu.au

K. Hasan, Department of Electrical Engineering, RMIT University, Australia.

(C) The Authors. Published by Blue Eyes Intelligence Engineering and Sciences Publication (BEIESP). This is an open access article under the CC BY-NC-ND license (http://creativecommons.org/licenses/by-nc-nd/4.0/) grid contingencies with their islanded operation independent of utility mains [3]. Networked micro grids help to avoid load shedding and meet excess load demand of one particular microgrid by sharing powers from the other neighboring microgrids in the network. Despite having numerous benefits, the expansion of networked microgrid has brought several technical challenges that are yet to be addressed efficiently with further research. The expansion has emerged many complexities since several controls are involved in functioning multiple microgrids as a coordinated network for the desired outcome. The bidirectional nature of power flow, frequent change in network topology, lesser electrical inertia and uncertainty in RES generation- all make the controlling of networked microgrid's operation to be differed from that of the traditional power distribution network. If not operated properly, networked microgrids suffer from poor load sharing. Moreover, sudden load changes in the system is prone to affect its voltage and frequency stability. Although in grid mode, microgrid's frequency and voltages are dictated by the main grid, these are of serious concern in microgrid's standalone mode which could lead the system fully unstable if remain ignored from proper regulation [4]. Thus, to provide satisfactory load sharing performance and power quality, a systematic control approach is required to be designed for the optimal coordination of multiple microgrids' power outputs and regulation of their voltages and frequency in both grid and islanded modes of operation.

To address the following challenges, a centralized control method has been proposed in [5] - [7]. In this approach, a single control unit is appointed centrally to gather all information from the sources and loads and determine the operation of the entire microgrid. However, the centralized control method demands for sophisticated communication links to transfer data and commands securely that are very expensive to implement. Moreover, single point risk of failure and compromise are also there [8]. Thus, this paper presents a decentralized cooperative control approach for the coordination of the microgrids that is more reliable and cheaper than the centralized control approach. Regardlessof the available communication signals, the proposed method applies control of droops, voltages and currents combinedly to work upon the local measurements that in turn assist the microgrids to share the increased loads instantly and be stabilized. Ref. [9] - [11] are some of the studies reported on the following decentralized control approach. However, the works presented in [10], [11] did not cover the microgrid's islanded mode of operation and were limited to be applicable only to the grid-connected microgrids.

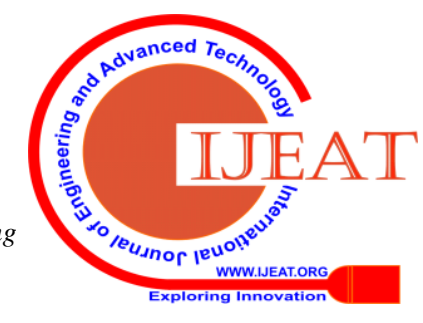


Moreover, to achieve the satisfactory control performance on power quality and load sharing, the parameters of the proposed controllers for power converters should be optimized before applying in the system. Ref. [12] - [14] reported the linearized model of small signal studies for the analysis of microgrid dynamics and optimization of control parameters. However, the control parameters obtained in this way only remains optimal at some specific system operating points due to the nonlinearities involved in microgrids, and the designed controllers fail to exhibit expected performance beyond the linearization- region around the vicinity of the system's equilibrium points. Again, it is very complicated to optimize the control parameters using traditional search heuristic methods of Ziegler Nichols [15], Pole Placement etc. as the networked system would have multiple PI controllers for multiple microgrids, and the result of tuning one controller would affect the tuned parameters of other controllers. Although Genetic Algorithm (GA) shows admissible performance in parameter optimization [16], Particle Swarm Optimization (PSO) algorithm outperforms it in diversification of the populations and escaping the local minima. Considering all these, a PSO based interlinked converter control algorithm is applied in this paper for the optimization of control parameters. The authors propose to allow the derivative-free algorithm to obtain fitness function from and apply solutions to the nonlinear microgrid simulation models directly. Thus, this paper achieves more practical results in terms of parameter optimization.

The remainder portion of the paper is organized as follows. Section 2 details the modelling of networked microgrid. Section 3 describes the proposed solution for comprehensive control of loads, frequency and voltages of the networked microgrid system. The algorithm framework of PSO that is used to optimize the control parameters is presented in the Section 4. Section 5 discusses the simulation results that validate the proposed solutions. Finally, Section 6 draws the conclusion on the following article.

\section{NETWORKED MICROGRIDS MODELLING}

The networked-microgrids is a concept of interconnecting a number of microgrid entities and integrating the features that different microgrids possess individually to the grid utility [17]. In the context of smart grid, if networked microgrids are designed and operated properly, these can serve as key elements for the black-start support of conventional units [4], longer emergency supply to the critical loads [18], better management of power uncertainties [19], and cost of energy (COE) minimization [3] by engaging clusters of locally and remotely distributed wind turbines (WTs) and solar photovoltaics (PVs). The interconnections of microgrids could be of various types based on the geographical locations and distribution system (DS) feeders (e.g. serial/parallel connections; single/multiple feeders etc.) [20], and analyzing all of them together would be complicated due to their different protection, control and communication settings. This paper considers a test bed system of interconnected microgrids on multiple DS feeders to study the overload dynamics. As depicted in Fig. 1, the test system is featured to address $\mathrm{x}$ number of microgrids, where each microgrid can have one or more points of common coupling (PCC) to exchange power with its neighboring microgrids and the utility grid.

As a controllable single entity, each microgrid will have its own DERs, ESSs, loads, transformers etc. within its defined electrical boundaries. The loads could be of different forms such as resistive, inductive and capacitive. The DERs could be modelled as wind farms having sets of induction generators and energy storage devices. These sources are electronically interfaced to the network using 3-level Voltage Source Inverters (VSI) along with coupling inductors and low

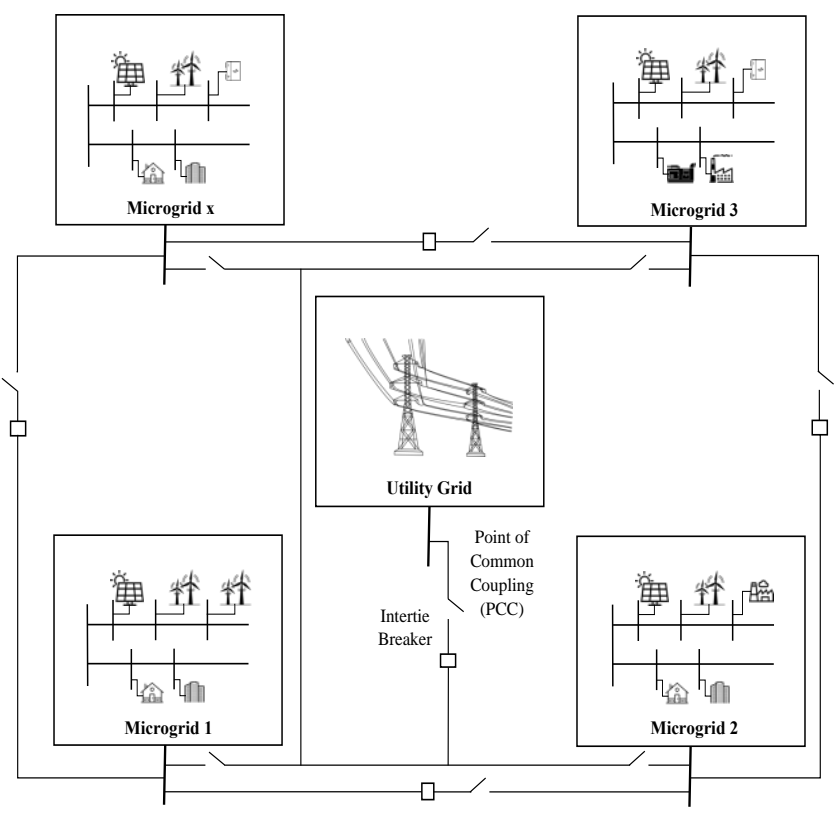

Fig. 1 Architecture of networked microgrid system studied

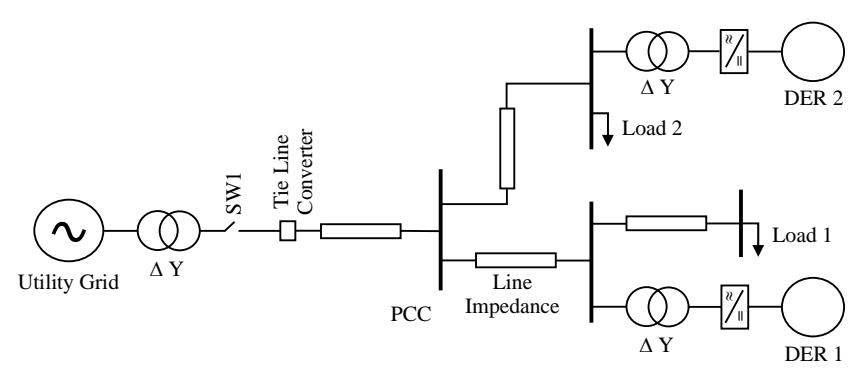

Fig. 2 Single line diagram of microgrid's connection to utility grid

pass LC filters that mitigate the high frequency switching harmonics [21]. Fig. 2 depicts the single line diagram of a microgrid's connection to the utility grid's distribution feeder through VSI interfaces. To align the slower control response of DERs with the response of VSIs, bulk DC link capacitances are employed to act as buffer energy storages between them. These capacitor banks should be sized enough, or additional DC batteries are required as energy storages to be fed from so that the performance of microgrid's power control does not degrade due to the delayed response of DERs, especially during the islanded operation of microgrid. Each energy system got both bidirectional ac-dc and dc-dc converters. The aim of dc-dc converters would be dispatching the generated power from wind turbines, whereas the ac-dc ones would control the voltages of different nodes of this large distribution network.

Blue Eyes Intelligence Engineering \& Sciences Publication

(C) Copyright: All rights reserved. 
To achieve the optimized performances of these converters, the authors have proposed to develop an interlinked framework to exchange information among the converters in a decentralized manner. With the following architecture of network, several mode of operations among the microgrids are possible. For instance, the topologies between MG1 and MG2 could be such that (a) Both MG1 and MG2 are operated in islanded mode, (b) Both MG1 and MG2 are operated in grid-connected mode, (c) MG1 remains grid-connected but MG2 is operated in islanded mode, (d) MG1 remains islanded but MG2 is operated in grid-connected mode, (e) MG1 is grid-connected and MG2 is interconnected to MG1, (f) MG2 is grid-connected, and MG1 is interconnected to MG2, and finally (g) MG1 and MG2 both are interconnected but islanded. To enable any of the following operation modes, the Distribution Management System (DMS) Operator will first determine the optimum power scheduling for the entire system, then command each microgrid to act upon accordingly. To connect the networked-microgrids to the main grid, a common connection of intertie breaker is provided. If a fault, natural disaster or maintenances take place at the main grid end, then the breaker is opened to isolate the network of microgrids and operate by itself.

\section{COMPREHENSIVE CONTROL DESIGN FOR POWER COORDINATION, AND VOLTAGE-FREQUENCY REGULATION}

To assist a networked microgrid on power distribution and regulation of voltage and frequency comprehensively, the proposed solution is designed to be an architecture of multiple outer and inner layers of different converter controlsdivided into primary, secondary and tertiary levels. Each layer has its own distinct feature to function. The layer of current control is responsible for the current flow regulation in the inductor filter circuit of the inverter. To regulate the voltage across the capacitors, the architecture also includes a voltage control function. The designed speed droopbased control layer performs the primary frequency regulation by acting upon the mismatch of the active power detected in the network as well as its availability of energy. A similar droop characteristic is also developed based on the relation between voltage and reactive power. Since the operating voltages and frequency of the system get changed from their nominal values with the load changes, these have to be restored to their previous operating states with the help of some secondary control functions. The power transfers among the DERs in a microgrid, as well as with the main grid and the neighboring microgrids are regulated using the mutual consensuses among the local controllers. The controllers are interlinked to exchange information with each other in a decentralized manner to adjust their reference points and redispatch the available DERs in the network such that no DER is overloaded at any point of time. The whole regulation process takes place until the system reaches its Nash equilibrium state. The key elements on achieving such effective control scheme and system operation are power electronic converters. In this section, all the control functions are discussed together required to operate the microgrids in islanded, grid-connected and networked modes of operation.

\section{A. Power Sharing Coordination Control}

The normal operation of any microgrid is such that it tries to fulfil its local demand from its local generation. If the microgrid's demand exceeds its local generation, then the required power is supplied by the main grid. Nevertheless, there are cases when the main grid fails to supply enough power, and the microgrid itself cannot increase its generation for maintenance issue or having less battery storage.

The power of the corresponding microgrid can then be supplied by the neighboring microgrids in the network [22]. This essential feature of networked microgrids can be brought into action by a DC-DC converter control of distributed generators (DG). The control strategy aims to re-dispatch the DERs located throughout the network and look for the neighboring microgrid having surplus generation to supply the affected microgrid's power mismatch. The controller follows the load power variation that decides the dispatch amount by reducing the reference error with the help of a compensator. The redispatch is decided by communicating with the local controllers of microgrids with an objective to minimize the overall cost of supply in the network [23]. Then based on the results obtained, the proposed controller instantly enables the sharing of active powers to redistribute among the microgrids by satisfying the network constraints, and the deficit power of the overloaded microgrid is supplied by the surplus generation of the other microgrid/s in the network as per the best choices available. Thus, each time a microgrid is overloaded in the network, the proposed controller adjusts the reference power and nullifies the imbalance through coordination among the microgrids without help of the main grid. This allows the additional hours of supply to the critical loads throughout the network during emergencies and improves the system reliability entirely.

Various droop control techniques such as active power frequency droop, reactive power - voltage droop, DC power voltage droop, and interlinking converter droop etc. can be used to perform the optimal load power sharing among the microgrids [18]. This paper applies the active power frequency and reactive power - voltage droop characteristics to be employed by the designed controllers. As designed in Fig. 3, the droops are implemented to emulate the behavior of conventional synchronous generators on their governor controls such that the frequency or voltage will decrease as the active or reactive power increases. Mathematically, the droop characteristics for each microgrid in the network can be formulated as follows.

$$
\begin{array}{r}
R^{M G x}=\frac{f_{o}-f}{P^{M G x}-P_{o}^{M G x}} \\
R^{M G x}=\frac{f_{o}-f}{P^{M G x}-P_{o}^{M G x}}
\end{array}
$$

where, $R^{M G x}$ is the positive coefficient that determine the slope of the speed droop curve of microgrid no. $M G x$ ( $x=$ $1,2,3, \ldots \ldots n), P^{M G x}$ and $f$ denote the value of active power output and frequency that are locally measured, and $P_{o}^{M G x}$ and $f_{o}$ are the reference values corresponding to the normal operating condition of the microgrid. Usually, the nominal value of the system frequency is selected to be the frequency reference. As it is depicted, due to any change in system operating condition, the amount of decrease (or increase) in frequencydetermines the amount of increase (or decrease) in the active power output of a DER. Thus, the DER will start shifting itsoperating point from point $\mathrm{X}$ to pointY along the speed droop curve shown in

Published By:

Blue Eyes Intelligence Engineering

\& Sciences Publication

(C) Convriaht: All riahts reserved.

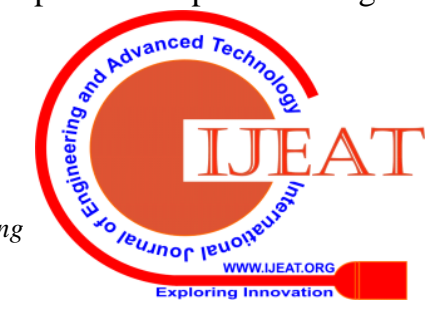


Fig. 3(b)until there is no power mismatchremained. Since the frequency value at the new operating point, $\mathrm{Y}$ will be different from its nominal value, a correctiveaction is required to be taken by sending load referencesignal so that the frequency is restored, and the systemperformance is stabilized at point Z. Hence, incorporating this reference offset signal,(1)can be rewritten as,

$$
\begin{gathered}
P^{M G x}=P_{o}^{M G x}+\frac{1}{R^{M G x}}\left(f_{o}-f+f_{\text {offset }}\right) \\
Q^{M G x}=Q_{o}^{M G x}+\frac{1}{M^{M G x}}\left(V_{o}^{M G x}-V^{M G x}+V_{o f f s e t}\right)
\end{gathered}
$$

A similar droop characteristic can also be developed between the parameters of voltage and reactive power according to (3), where $M^{M G x}$ is the droop slope. $V^{M G x}$, $Q^{M G x}$ and $V_{o}^{M G X}, Q_{o}^{M G x}$ are the locally measured and the reference signals for voltage

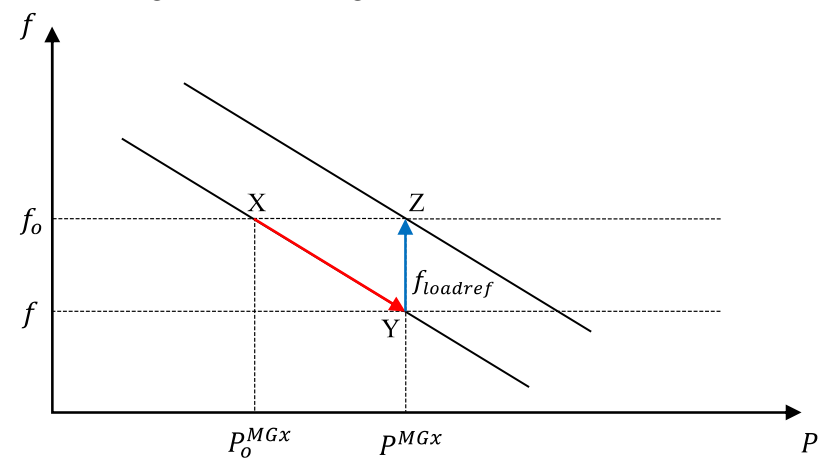

(a) Droop relation between active power and frequency

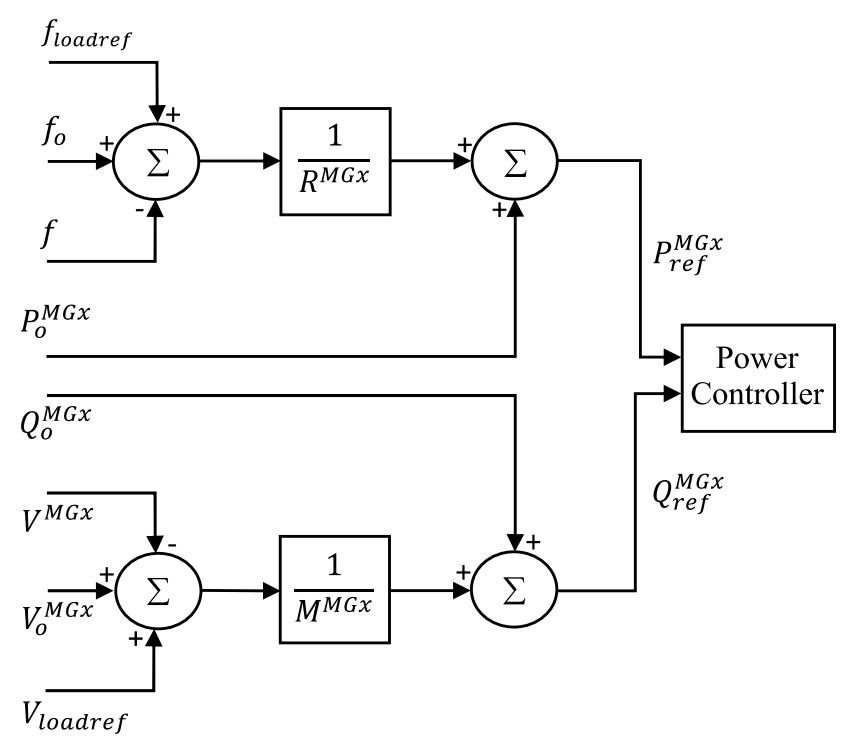

(b)Block diagram of droop controls

Fig. 3 Active power - frequency and reactive power voltage droop controls

and reactive power respectively. $f_{\text {offset }}$ and $V_{\text {offset }}$ are the load reference signals sent to clear the offsets in frequency and voltage from their nominal values.

The droop controllers of the microgrids will act in a decentralized way to bring tight adjustments over the frequency and bus voltages throughout the network and assign different power sharing among the microgrids by setting different values for their droop constants.

\section{B. VSI Inverter Control}

The sudden load change in the networked system will drive it to experience deviations in both voltages \& frequency. If any of these is ignored in regulating, then the whole system will become unstable. In the grid-connected mode, the voltage and frequency regulation is strongly supported by the main grid. However, there will be events of natural disasters or maintenance issues due to which the maingrid has to be disconnected fully from the networkedmicrogrids and can no longer provide frequency or voltage support. Therefore, to mitigate the effects of overloading event, the proposed control solution must be comprehensive in not only performing the power exchange among the microgrids to nullify the imbalance but also enabling the networked microgrids to stabilize voltages and frequency by itself within the acceptable margins. To perform such restorations, inner loops of controllingvoltage \& current along with outer power loops having droops are designed.

At first, using Park transformation, the three phase values in time domain are converted from abc reference frame to dq0 rotating reference frame. It will simplify the calculation since the converted values will be constant, and the last component of dq0 coordinate becomes zero when the load demand is balanced. After that, the following loops are developed in d-q axes separately such that both the active and reactive power controls are decoupled into $d$ and $q$ components through per unit system representation.

As shown in Fig. 4, in d-control frame, an active power control loop is designed such that the locally measured power $P^{M G x}$ will track the reference power signal $P_{r e f}^{M G x}$ generated by the speed droop controller. The generated error is then fed into a PI controller to obtain reference current $i_{d \_r e f}^{M G x}$ for the current controller. The proportional and integral coefficients of this controller denoted as $K_{p 1}$ and $K_{i 1}$ are optimized using PSO algorithm later in this paper. Using the obtained reference value, a sufficient amount of inverter current need to be extracted from the system to inject in d-axis through the inverter which will stabilize the system voltages in turn.A current controller is thus implemented as shown in Fig. 5using another PI compensator with gains $K_{p 2}$ and $K_{i 2}$ to helpthe locally measured current $i_{d}^{M G x}$ to followthe inverter output current reference $i_{d}^{M G} r e f$ and generate reference voltage of inverter $v_{d}^{M G X}$ ref . Furthermore, in the inner loop, the locally measured voltages are transformedfrom abc frame to dq frame in order to compare with the reference voltage. Again, a similar control frame isimplemented in q-axis. This time the difference in reactivepower loop is used based on the voltage droop slope of $M^{M G x}$. Here, the reference for the current controller is set as $i_{q_{-} r e f}^{M G x}$ for current injection in q-frame to generate reference voltage $v_{q_{\text {_r }} \text { ref }}^{\text {MGx }}$ for the inverter circuit. Finally, both the $\mathrm{d} \& \mathrm{q}$ components of the reference voltage, $v_{d_{-} \text {ref }}^{M G X}$ and $v_{q_{-} \text {ref }}^{\text {MGx }}$ are transformed back into abc

Published By:

Blue Eyes Intelligence Engineering \& Sciences Publication 


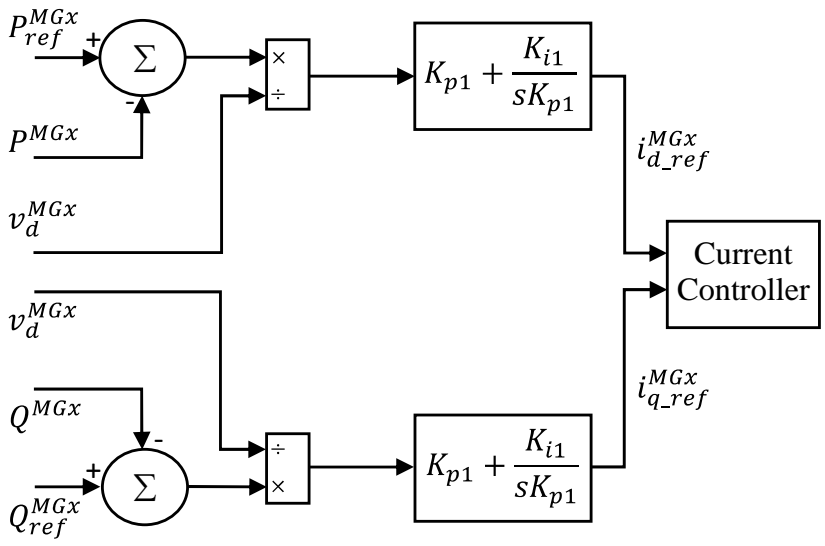

Fig. 4Block diagram of power controller

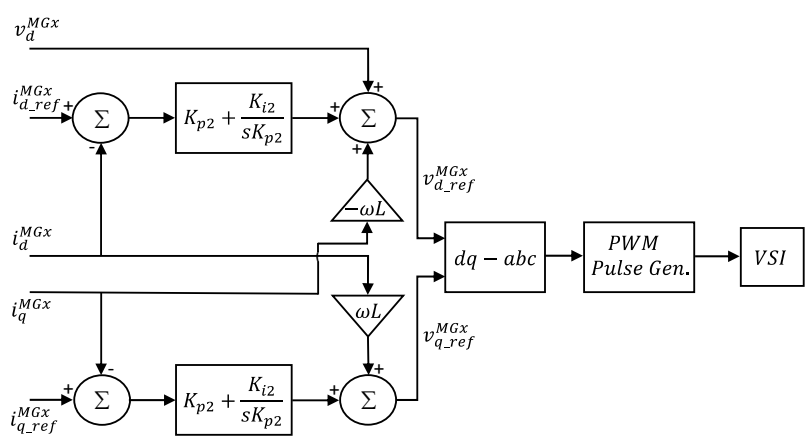

Fig. 5Block diagram of current controller

frame and then converted into PWM gating signals to be extracted as duty cycles to feed into the switches presented in the system to maintain the system stability during any load variation.

In this paper,the authors have preferred using 3-level VSIto 2-level VSI so that the output harmonics can be sufficiently reduced by the interfaced inductor without the help of capacitors, and the circuit becomes simpler [21].The proposed three phase 3-level VSI is a nine switches topology, which is designed by connecting the source with the full bridge power switches of classical 2-level inverter through an auxiliary circuit of three bidirectional switches. As shown in Fig. 6, there are 2 isolated units of $\mathrm{H}$-bridge circuits along with the two bulk top and bottom capacitor banks $\left(\mathrm{C}_{\mathrm{Top}}\right.$ and $\left.\mathrm{C}_{\text {Bottom }}\right)$ in order to function the inverter. The main 2-level inverter represented by $\mathrm{Q}_{1}-\mathrm{Q}_{6}$ operates with a switching frequency equals to the $50 \mathrm{~Hz}$ of line-frequency, whereas the auxiliary circuit of bidirectional power switches denoted by $\mathrm{S}_{1}-\mathrm{S}_{3}$ allow energy flowing in both the directions with a switching frequency of $100 \mathrm{~Hz}$ that is double of the line-frequency. Using such a multilevel VSI will result in a significant suppression of harmonics distortion in the outputs of inverter compared to that achieved with a conventional 2-level VSI. The following equation can be written to represent the VSI output circuit that is modelled with an equivalent inductance and resistance of $L_{s}$ and $R_{s}$.

$$
\begin{gathered}
L_{s} \frac{d}{d t}\left[\begin{array}{l}
i_{a}^{M G x} \\
i_{b}^{M G x} \\
i_{c}^{M G x}
\end{array}\right]+R_{s}\left[\begin{array}{l}
i_{a}^{M G x} \\
i_{b}^{M G x} \\
i_{c}^{M G x}
\end{array}\right]=\left[\begin{array}{c}
v_{s a}^{M G x} \\
v_{s b}^{M G x} \\
v_{s c}^{M G x}
\end{array}\right]-\left[\begin{array}{l}
v_{a}^{M G x} \\
v_{b}^{M G x} \\
v_{c}^{M G x}
\end{array}\right] \\
\frac{d}{d t}\left[\begin{array}{l}
i_{a}^{M G x} \\
i_{b}^{M G x} \\
i_{c}^{M G x}
\end{array}\right]=\frac{R_{s}}{L_{s}}\left\{\frac{1}{R_{s}}\left(\left[\begin{array}{l}
v_{s a}^{M G x} \\
v_{s b}^{M G x} \\
v_{s c}^{M G x}
\end{array}\right]-\left[\begin{array}{l}
v_{a}^{M G x} \\
v_{b}^{M G x} \\
v_{c}^{M G x}
\end{array}\right]\right)-\left[\begin{array}{l}
i_{a}^{M G x} \\
i_{b}^{M G x} \\
i_{c}^{M G x}
\end{array}\right]\right\}
\end{gathered}
$$

where, the voltages and currents are measured in abc frame, and the inverter outputs are denoted with the 's' subscript. We know that the Park's transformation can be formulated as

$$
\left[\begin{array}{l}
d \\
q \\
0
\end{array}\right]=\frac{2}{3}\left[\begin{array}{ccc}
\cos \theta & \cos \left(\theta-\frac{2 \pi}{3}\right) & \cos \left(\theta+\frac{2 \pi}{3}\right) \\
-\sin \theta & -\sin \left(\theta-\frac{2 \pi}{3}\right) & -\sin \left(\theta+\frac{2 \pi}{3}\right) \\
\frac{1}{2} & \frac{1}{2} & \frac{1}{2}
\end{array}\right]\left[\begin{array}{l}
a \\
b \\
c
\end{array}\right]
$$

where, $\theta=\omega t=\omega_{s} t+\theta_{0}$ is the angle between a and $\mathrm{d}$ axes with $\omega$ rotational speed of $d-q$ reference frame. Thus, the transformation of (5) can be expressed in d-q frame as

$$
\frac{d}{d t}\left[\begin{array}{l}
i_{d}^{M G x} \\
i_{q}^{M G x}
\end{array}\right]=\frac{1}{L_{s}}\left(\left[\begin{array}{l}
v_{s d}^{M G x} \\
v_{s q}^{M G x}
\end{array}\right]-\left[\begin{array}{l}
v_{d}^{M G x} \\
v_{q}^{M G x}
\end{array}\right]\right)+\left[\begin{array}{cc}
-\frac{R_{s}}{L_{s}} & \omega \\
-\omega & -\frac{R_{s}}{L_{s}}
\end{array}\right]\left[\begin{array}{l}
i_{d}^{M G x} \\
i_{q}^{M G x}
\end{array}\right]
$$

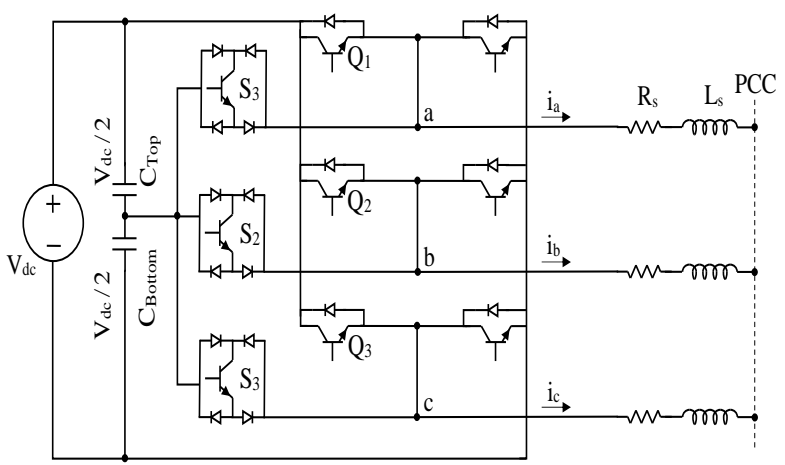

Fig. 6Proposed 3-level VSI model with nine switches

Then the current controllers having PI controller of gains $\mathrm{K}_{\mathrm{p} 2}$ and $\mathrm{K}_{\mathrm{i} 2}$ generate reference voltages of inverters as follows.

$$
\begin{aligned}
{\left[\begin{array}{l}
v_{s d_{\_} r e f}^{M G x} \\
v_{s q_{-} r e f}^{M G x}
\end{array}\right]=\left[\begin{array}{cc}
K_{p 2} & 0 \\
0 & K_{p 2}
\end{array}\right]\left[\begin{array}{l}
i_{d_{-} r e f}^{M G x} \\
i_{q_{-} r e f}^{M G x}
\end{array}\right] } \\
+\left[\begin{array}{cc}
-K_{p 2} & -\omega L_{s} \\
\omega L_{s} & -K_{p 2}
\end{array}\right]\left[\begin{array}{c}
i_{d}^{M G x} \\
i_{q}^{M G x}
\end{array}\right]+\left[\begin{array}{c}
v_{d}^{M G x} \\
0
\end{array}\right] \\
+\left[\begin{array}{cc}
\frac{K_{i 2}}{K_{p 2}} & 0 \\
0 & \frac{K_{i 2}}{K_{p 2}}
\end{array}\right]\left[\begin{array}{l}
X_{d} \\
X_{q}
\end{array}\right]
\end{aligned}
$$

Where, $K_{p 2} / K_{i 2}=T_{i 2}$ is the integral time constant for PI controller of the current controller to reset and the circuit reactance is defined according to (9).

$$
\left[\begin{array}{l}
X_{d} \\
X_{q}
\end{array}\right]=\int\left[\begin{array}{l}
i_{d_{-} r e f}^{M G x}-i_{d}^{M G x} \\
i_{q_{-} \text {ref }}^{M G x}-i_{q}^{M G x}
\end{array}\right] d t
$$

\section{CONTROL PARAMETERS OPTIMIZATION}

This section yields the optimization process of the parameters used for the designed controllers. The authors propose to use PSO algorithm to optimize the control parameters. Introduced by Kennedy and Eberhart in 1995, the algorithm is implemented based on the social behaviors of bird flocking in searching foods [24]. Since there are multiple PI controllers in the system, the tuning of one controller would affect the tuning of others, and thus the traditional

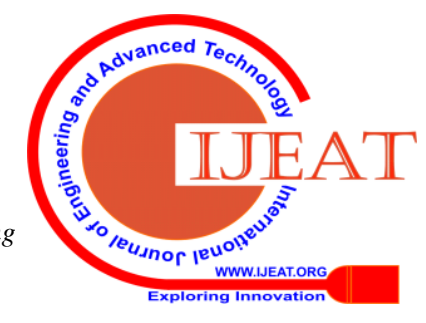


methods like Ziegler Nichols, Pole Placement etc would make the tuning process more complicated. Besides, the optimization algorithms like GA (Genetic Algorithm) and PSO provides much freedom in defining the fitness function to be optimized. However, PSO performs better than GA in finding the globally optimal solution as it diversifies the solution particles with a term of stochastic velocity.

\section{A. Implementation of Algorithm}

The algorithm is initialized with a group of randomly generated solutions (particles) in the problem search space. The group is defined as a set of population, and each single particle in the population set gets its own fitness values evaluated through a fitness function defined based on the problem objective. The particles resemble birds in the context of above example and have velocities by which their movements are directed towards the optima vicinity of the solution space. The algorithm then moves into the next iteration by updating each particle with two 'best' fitness values defined as: (1) PBest, which corresponds to the best fitness solution in the current population set achieved through its local experience; and (2) GBest, that corresponds to the global best solution tracked so far by the collective experience of the algorithm. To update the velocity and position of a particle in the algorithm, the following search rules are applied as in (10)and (11).

$$
\begin{gathered}
V_{i}(k)=\omega V_{i}(k)+\varphi_{1} r_{1}\left[X_{\text {PBest }_{i}}-X_{i}(k-1)\right] \\
+\varphi_{2} r_{2}\left[X_{\text {GBest }_{1}}-X_{i}(k-1)\right] \\
X_{i}(k)=X_{i}(k-1)+V_{i}(k)
\end{gathered}
$$

where, $X_{i}(k)$ and $V_{i}(k)$ is the position vector and velocity vector of the $i$-th particle at the iteration no. $k$, along with inertia weight of $\omega$, two positive constants of $\varphi_{1} \& \varphi_{2}$ and the random numbers of $r_{1} \& r_{2}$ generated in the range of $[0,1]$. $X_{\text {PBest }_{i}}$ is the best (population best) position of the $i$-th particle achieved through its individual experience, whereas $X_{\text {GBest }}$ is the best (global best) position of any particle achieved so far based on the algorithm's overall experience.

As it can be seen, the movement of each particle is determined by the three directional terms of its velocity mentioned in (10). The first term drives the diversification with the random movements of particles towards diverse areas of the solution space. The second and third ones represent intensification such that the particle movements are drawn towards the vicinities of the local best and global best positions. Therefore, PSO is able to avoid converging into the local minima unlike the other derivative based algorithms.

To enhance the algorithm performance, the value of $\omega$ is determined by the dynamic inertia method of (12) rather than just setting it randomly to a constant value [25]. This will provide the particle-movements high velocities for the early search and then low velocities to the later search in the surrounding zones of the best positions achieved.

$$
\omega=\omega_{\max }-\frac{k\left(\omega_{\max }-\omega_{\min }\right)}{N}
$$

where, $N$ is the total number of iterations, with $\omega_{\min }=0.4$ and $\omega_{\text {max }}=0.9$.

Moreover, to satisfy the search space constraints, if any $j$-th element of an $i$-th particle is found to cross its boundary limit of $X_{j}^{\text {limit }}$, then its position and velocity are directed to be updated according to (13) with a random number, $r$ generated between $[0,1]$.

$$
X_{i j}(k)=X_{j}^{l i m i t} ; \quad V_{i j}(k)=-r \times V_{i j}(k-1)
$$

The scope of PSO in this work arouse out of the requirement of selecting proper control parameters for the microgrids to achieve their optimal performances on sharing loads and maintaining quality power. The parameters that are set as particles in this algorithm framework to be optimized in achieving the minimum fitness function are selected as follows.

$$
X=\left[K_{p 1}, K_{i 1}, K_{p 2}, K_{i 2}, R_{B}, M_{B}\right]
$$

where, $K_{p 1}, K_{i 1}, K_{p 2}$ and $K_{i 2}$ are the proportional and integral coefficients of PI controllers mentioned in the previous section. The parameters of $R_{B} \& M_{B}$ are used to determine the values of droop slopes such that

$$
R^{M G x}=r^{M G x} \times R_{B} ; \quad M^{M G x}=m^{M G x} \times M_{B}
$$

where, $r^{M G x} \& m^{M G x}$ denote the changing ratios of active power outputs and reactive power outputs of the different microgrids' DERs respectively. These values are not constant and changed in different stages of network-operation as per requirement.

The control parameters in (14) and (15) are selected as the particles in relevance to the problem statement of this paper. With the help of a fitness function, each particle is given a fitness value and directed to fly with a velocity through the problem space by following the fittest particles achieved so far. In this way, the whole algorithm learns from the scenario and finds out the optimum solutions by updating generation after generation. The flowchart of the overall PSO algorithm is given in Fig. 7.

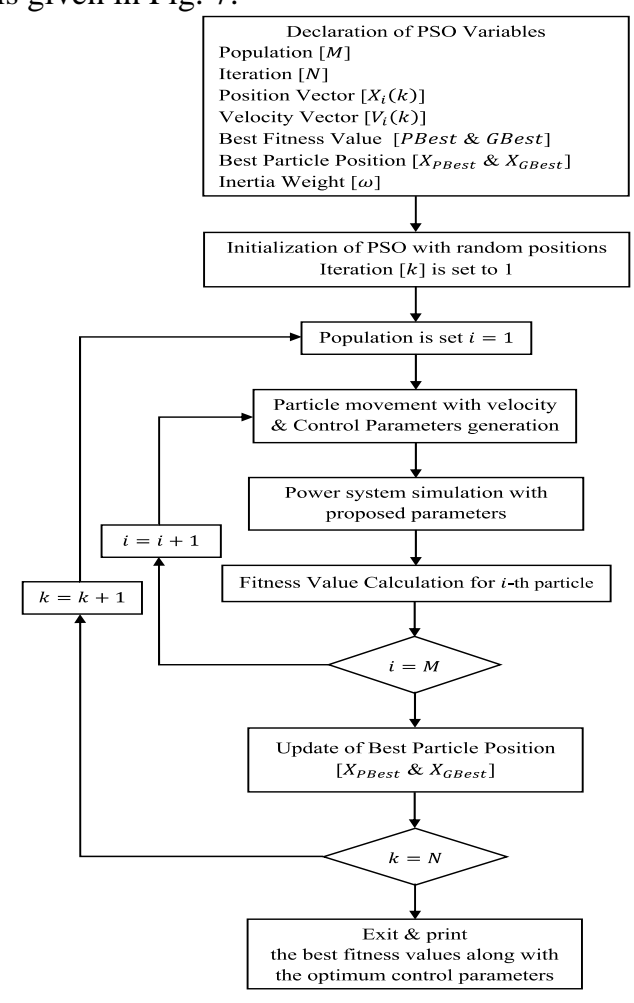

Fig. 7PSO algorithm flowchart in parameters optimization

Published By:

Blue Eyes Intelligence Engineering \& Sciences Publication 


\section{B. Formulation of Fitness Function}

The fitness function defined for the optimization problem would be the only interface through which the physical test microgrid disconnection or any load change occurs in the network. In each stage, there are three performance indices that are selected to be assessed such as load sharing performance, bus voltage performance and frequency performance. For the evaluation of the bus voltages and frequency performance, the error indices that are included in the fitness function are defined as the differences from theirnominal values of $1 \mathrm{pu}$ and $50 \mathrm{~Hz}$ respectively. On the other hand, to evaluate the load sharing performance criteria, the error index is defined as the difference between the locally measured active power values and the set power references. These referencesare set as the output power references of DERs in the grid mode of operation, whereas in any other operation modes, the references are set depending on the amount of the load demands present in the network. Finally, to ensure power quality, the voltage and frequency variations are maintained within their threshold margins of \pm $1 \mathrm{~Hz}( \pm 0.02 \mathrm{pu})$ and \pm 0.1 purespectively throughout the simulation process. Thus, two more elements are added in order to cost the fitness function penalty on violating the following constraints set for the RMS bus voltages and the system frequency.

The authors propose to express the following error indices in the form of ITAE (Integrated Time Absolute Error) as defined in (16)since it delivers the more realistic value than the other three types of error assessment functions namely IAE (Integrated Absolute Error), ISE (Integrated Squared Error), and ITSE (Integrated Time Squared Error) [26].

$$
\text { ITAE }=\sum_{t}^{t} t\left|E\left(X_{t}, t\right)\right|
$$

So, the overall fitness function to be minimized can be formulated as follows.

$$
\begin{gathered}
F=\sum_{j=1}^{n} I T A E^{j}+P N L T Y^{v}+P N L T Y^{f} \\
F=\sum_{t^{j}=t_{o}^{j}}^{t_{f}^{j}}\left(t^{j}-t_{o}^{j}\right) \times\left|E^{j}\left(t^{j}\right)\right|+C^{v} \lambda^{v}+C^{f} \lambda^{f} \\
\left|E^{j}(t)\right|=W^{j}\left[\begin{array}{l}
\left|\Delta P^{j}(t)\right| \\
\left|\Delta V^{j}(t)\right| \\
\left|\Delta f^{j}(t)\right|
\end{array}\right]
\end{gathered}
$$

where, the functions of 'ITAE' evaluate the performances of the network in terms of active power, voltage and frequency for all the ' $j=1,2,3, \ldots . . n$ ' stages of a simulation distinguished by the events like grid disconnection, microgrid disconnection, load change etc. The functions of ' $P N L T Y$ ' deliver the penalty scores on the violation of voltages and frequency constraints as aforementioned. ' $t$ ' is the sampled simulation time for the calculation of the performance indices of active power, voltage and frequency in each stage with $t_{o} \& t_{f}$ as the starting and ending points of the sampled period. ' $|E(t)|$ ', as defined in (19), is the absolute error matrix with three elements that represent the error between locally measured values and the reference values for the three performance indices of active power, voltage and frequency separately. As it can be noticed, to make the performance index of active power more important in the assessment process than the other 2 indices of voltages and frequency, it is weighted twotimes larger with the inclusion of the weight matrix of $W=\left[\begin{array}{lll}2 & 1 & 1\end{array}\right]$. The voltage \& frequency parameters are rather regulated with the penalty constant of ' $C$ ' having a large value. The penalty function also contains ' $\lambda$ ' as a constraint violation index such that it is equal to 1 if the constraint of RMS voltage variation of $\pm 0.1 \mathrm{pu}$ or frequency variation of $\pm 1 \mathrm{~Hz}$ is violated. Otherwise, it is equal to 0 to nullify the effect of the penalty functions upon the fitness function.

\section{CASE STUDIES AND RESULTS ANALYSES}

The effectiveness of the proposed control strategy is verified through PSCAD simulation on a similar test bed system as of Fig. 1. A network of three microgrids interconnected with the main grid via three individual VSI inverters is simulated. As mentioned earlier, the VSIs are selected as 3-level VSIs having PWM switching through insulated-gate bipolar transistors (IGBT). The number of the main power diodes and power switches for the inverter circuit are chosen as 12 and 9 respectively. Each of the three bidirectional switches gets 4 fast diode rectifiers along with 1 IGBT. The selected DS feeder is of $13.8 \mathrm{kV}$ and 3 phase step-up transformers are used to connect the DERs with it. The DERs are rated as 3.2 MW, 1.7 MW and 2.3 MW. The initial load demands of microgrids no. 1, 2 and 3 are chosen to be 2.3 MW, 1.5 MW and 1.75 MW respectively. The nominal frequency of the system is set as $50 \mathrm{~Hz}$. The microgrids can be operated in both grid or islanded mode with the help of an inter-tie breaker common connection that is provided to isolate the microgrids from the main grid during natural disasters, maintenances etc.

To optimize the control parameters with PSO algorithm, the power system along with the presented converter control is simulated in PSCAD platform, while the algorithm is coded in m-file of MATLAB. Once a MATLAB version is linked in PSCAD for use, a new MATLAB block is developed and inserted in the DRAFT palette of PSCAD. The necessary codes of PSO are then written as user input in the text-editor shell of the graphical block icon. The population and iteration numbers of the algorithm are set as 20 and 35 respectively. This means that the system would be simulated 700 times in PSCAD by calling the MATLAB function. To repeat the simulation for such multiple times, the Multiple-Run component of PSCAD library is used. The microgrids are assumed to have identical PI controllers for this study. Thus, two proportional coefficients and two integral coefficients would be optimized for the available power and current controllers. Each time some values are suggested by the algorithm for the coefficients, the PSCAD software would run the simulation and the system performances are assessed by the fitness function. The global best position is supposed to update in every 20 simulations as the number of particles in each iteration is 20 .

The simulation is directed to comprise of four cases to depict the reliability of the networked microgrids with the proposed control strategy. At first, all the microgrids remain connected to the main grid in the simulation process.

Published By:

Blue Eyes Intelligence Engineering \& Sciences Publication

(C) Convriaht: All riahts reserved. 
Then the networked-microgrids is simulated by opening the breaker and disconnecting the main grid. Thirdly, an overloading event is triggered to happen in the microgrid no. 2. Finally, the microgrid 3 is also isolated from the network. Thus, each simulation would contain four evaluations on the performances of the network's active power sharing, voltages and frequency. Each simulation would also have two penalization factors to be measured by the fitness function. The searching for the optimal values of control parameters through the algorithm are depicted in Fig. 8 and Fig. 9. Fig. 8shows that the fitness function converges at the end of the iterations. Only those control parameter values which show lower fitness values than the set value of constant, $C=500 \mathrm{in}$ (18) remain appropriate candidate sets for the controllers considering the fact that the voltages and frequency constraints are not violated. As it can be seen from these figures, such candidates are first found to be appeared after $13 \times 20=260$ number of simulations.

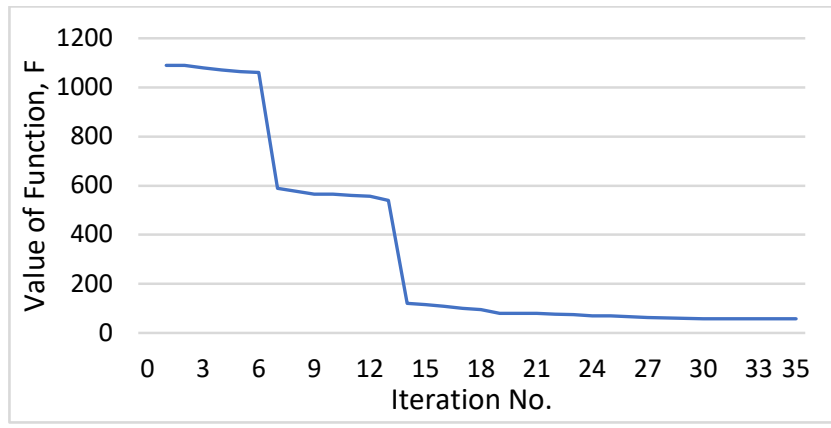

Fig. 8 Value of Fitness Function at each Iteration Stage

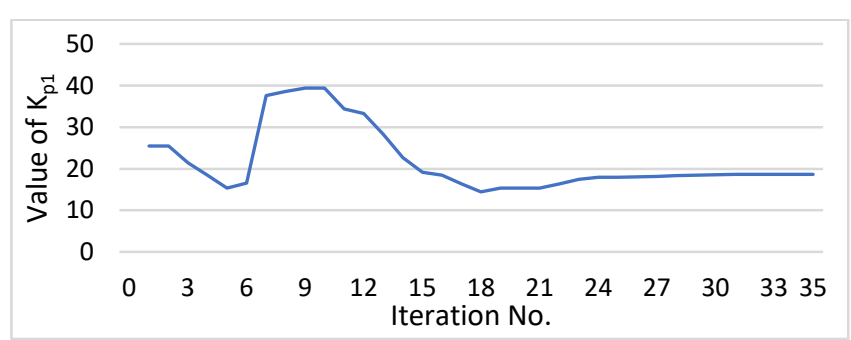

(a) Control Parameter, $K_{p 1}$

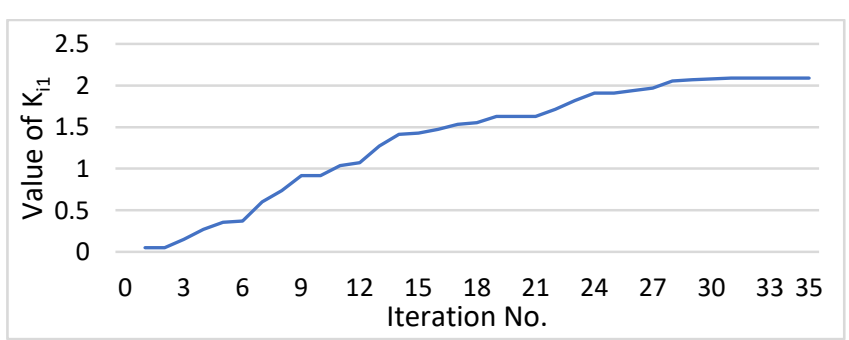

(b) Control Parameter, $K_{i 1}$

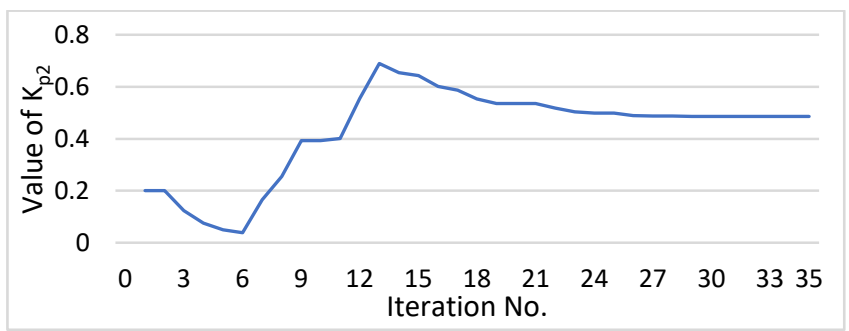

(c) Control Parameter, $K_{p 2}$

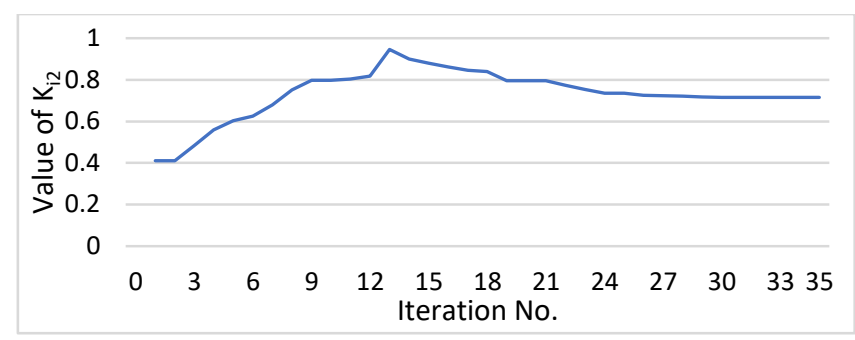

(d) Control Parameter, $K_{i 2}$

Fig. 9Control Parameters Optimization with PSO

Table- I lists the most optimal values of the control parameters that the algorithm obtains for the case studies. The performances of the following networked-microgrids simulated with the optimized control parameters are shown in Fig. 10, and Fig. 11. The results show that the proposed solution is comprehensive not only in exchanging poweramong the microgrids to nullify the network's imbalance, but also in stabilizing the network's voltages and frequency within the acceptable margins. The results are discussed sequence by sequence of the simulation as follows.

From $t=0.0 \mathrm{~s}$ to $\mathrm{t}=0.3 \mathrm{~s}$ is of less significance and kept to initialize the simulation with the data stored in the snapshot file. The network of the microgrids remains grid-connected without any power generation.

At $\mathrm{t}=0.3 \mathrm{~s}$, the generators are controlling the microgrids' output power as per the preset values. Here, MG1 generates 2.9MW in the network, whereas the generations of MG2 and MG3 are set as $1.7 \mathrm{MW}$ and $2.25 \mathrm{MW}$ respectively (see Fig. $10(\mathrm{~b}))$. The total load demand of the network is set as 5.55MW such that loads of MG1, MG2 \& MG3 are 2.3MW, 1.5MW and 1.75MW (see Fig. 10(a)). Since the network remains grid-connected, the surplus amount of generated power, 1.3MW is exported to the main grid as shown in Fig. 10 (c). The voltages and frequency of the network are strongly supported by the main grid and stay at their nominal values of $1 \mathrm{pu}$ and $50 \mathrm{~Hz}$ respectively which can be seen in Fig. 11(a) and Fig. 11(b).

At $\mathrm{t}=0.6 \mathrm{~s}$, the networked microgrids are islanded from the main grid by opening the inter-tie breaker assuming that a contingency is happened at the main grid end. Sensing the grid isolation, the controllers decrease the generations of three microgrids to match their existing demands of 2.3 MW, 1.5 MW and 1.75 MW. The change ratio of the output power is seen as $M G 1: M G 2: M G 3=0.6: 0.2: 0.5$ or, 1: $0.333: 0.833$.

Table I The optimum values of control parameters for the selected networked MG system

\begin{tabular}{cc}
\multicolumn{2}{c}{ the selected networked MG system } \\
\hline Control Parameter & Optimum Value \\
\hline$K_{p 1}$ & 18.616845 \\
$K_{i 1}$ & 2.090443 \\
$K_{p 2}$ & 0.485374 \\
$K_{i 2}$ & 0.715324 \\
$R_{B}$ & 0.451349 \\
$M_{B}$ & 3.820154 \\
\hline
\end{tabular}

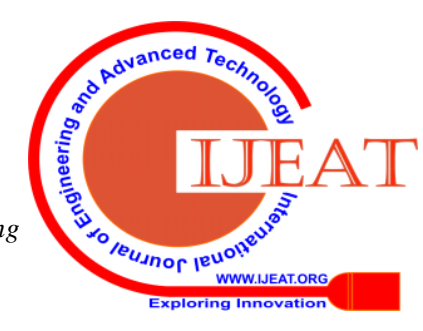




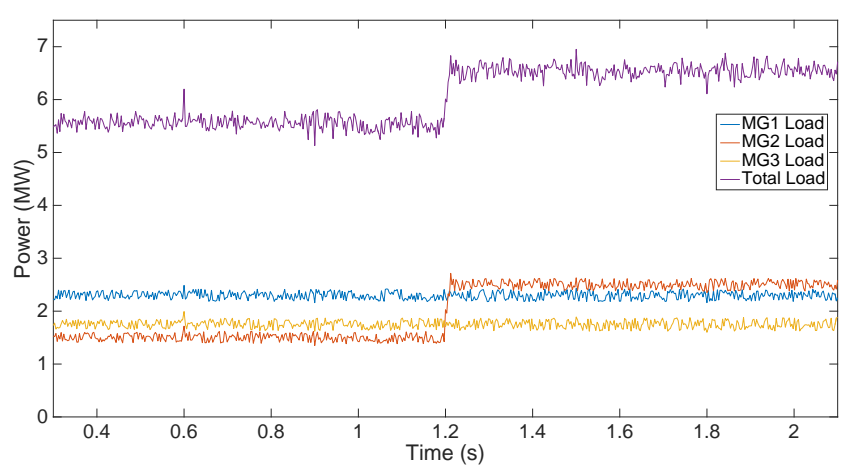

(a)Load demands of MG1, MG2 and MG3

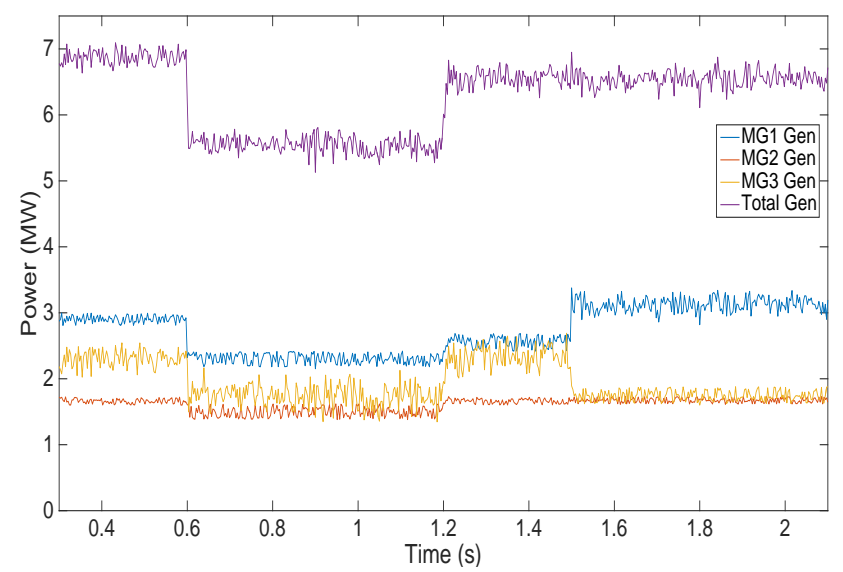

(b)Generation outputs of MG1, MG2 and MG3

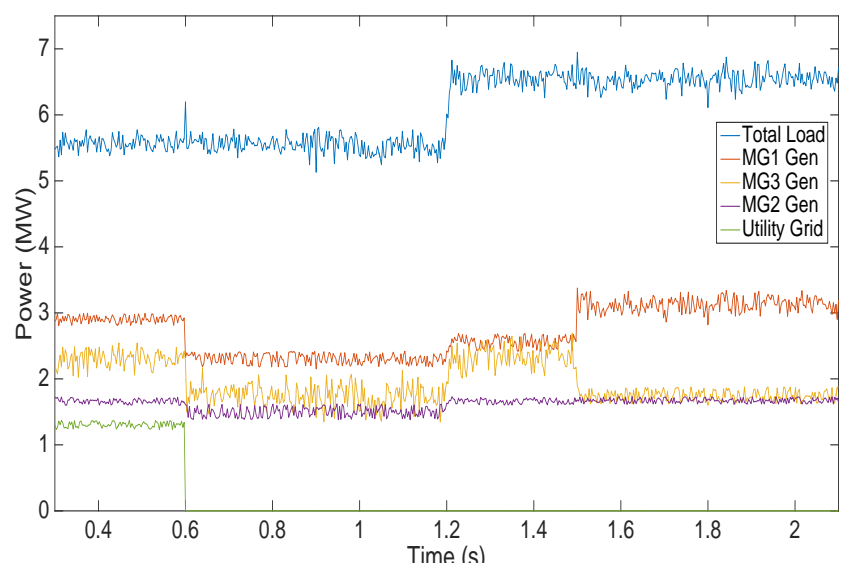

(c)Active power share of MG1, MG2 and MG3 with each other and utility grid

Fig. 10Responses of the networked-microgrids with the proposed control solution towards grid-disconnection, load change and microgrid isolation

This change ratio is automatically realized by the three droop controllers by setting $r^{M G X}$ as $1 \%: \frac{1}{0.333} \%: \frac{1}{0.833} \%$ or, $0.010: 0.030: 0.012$. The actions of the droop controllers would result in operating point changes of system frequency and voltages.

At $t=0.9 \mathrm{~s}$, load reference signals are sent by the controllers for restoring the voltage and frequency close to $1 \mathrm{pu}$ and $50 \mathrm{~Hz}$, which are their nominal values as in Fig. 11 (a) \& (b).

At $\mathrm{t}=1.2 \mathrm{~s}$, an overloading event is triggered to happen in MG2, and its demand is increased by 1 MW (see Fig. 10(a)). Although MG2 increases its generation to mitigate the extra demand, its real power reference cannot be changed beyond its maximum 1.7MW rating. Moreover, the utility grid being disconnected cannot supply the affected microgrid. Therefore, in this case, the neighboring microgrids of MG1 and MG3 are requested through the proposed controllers to supply the rest of the extra demand. The two microgrids then increase their real power outputs instantly following their droop characteristics. As it is reflected in Fig. 10(b), the microgrids decide redispatch on their generators through the controllers at $\mathrm{t}=1.2 \mathrm{~s}$ to support the overload. Here, as per the assumptions, MG3 is first prioritized to increase its generation till its maximum limit of 2.3 MW, then MG1 supplies the rest of the extra demand.

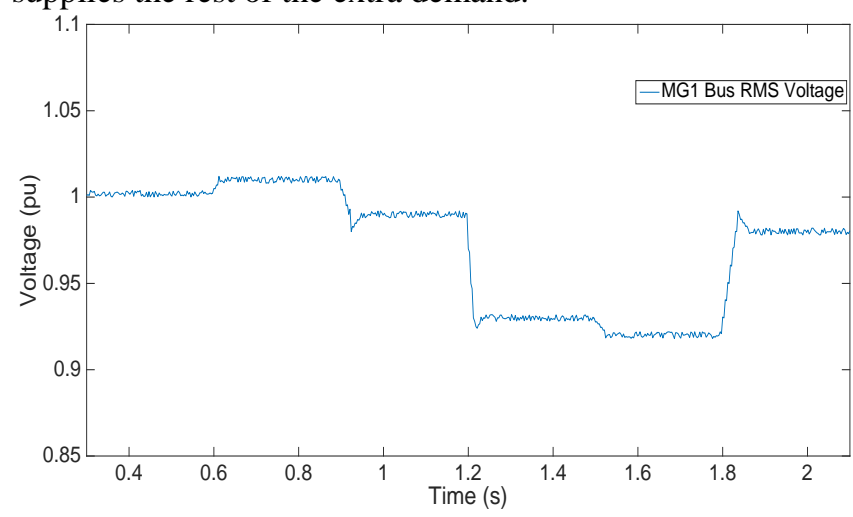

(a)RMS bus voltage at MG1

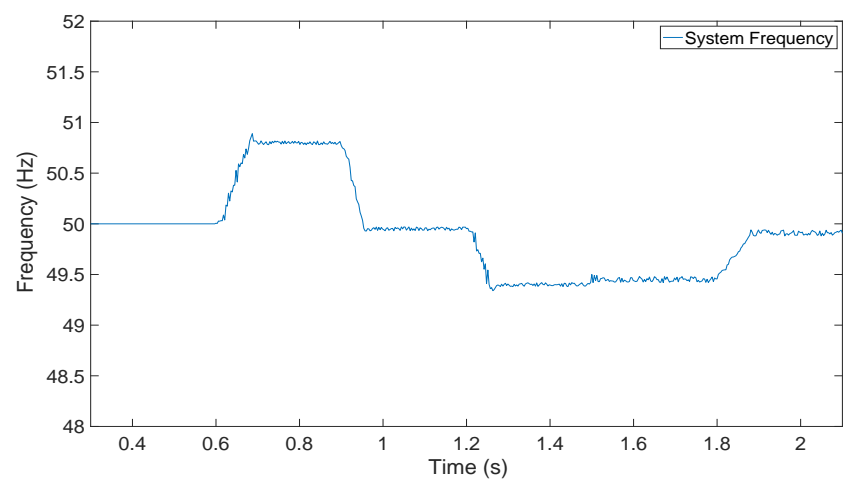

(b)System frequency

Fig. 11Voltage and frequency responses of the networked-microgrids with the proposed control solution

At $\mathrm{t}=1.5 \mathrm{~s}, \mathrm{MG} 3$ is assumed to be disconnected from the MG1 and MG2 by opening the switch at its PCC due to another contingency. Thus here, the additional power demand of MG2 is shared by MG1 only, and MG3 generates power only to supply its own load as it can be seen in Fig. 10(c).

Fig. 10 (a), (b) and (c) show how the controllers assist the microgrids in sharing active powers in the network in different situations and handling overloads. However, sudden load change would drive the networked-microgrid to experience voltage deviations as well as frequency deviations. Since in islanded modes of operations, the utility grid can no longer provide frequency or voltage supports, the proposed controllers must have tight controls over the voltages and frequency during these load variations. The voltage profile and system frequency shown in Fig. 11 (a) and (b) depict that these remain within their acceptable margins of $\pm 0.1 \mathrm{pu}$ and $\pm 1 \mathrm{~Hz}$, and the stability of the system is ensured throughout the process with the employment of the following controllers. Finally, at $\mathrm{t}=1.8 \mathrm{~s}$, it can be seen that load reference signals are sent for the second time in the simulation to restore the network voltages and frequency close to their nominal values.

Published By:

Blue Eyes Intelligence Engineering \& Sciences Publication

(C) Convriaht: All riahts reserved.

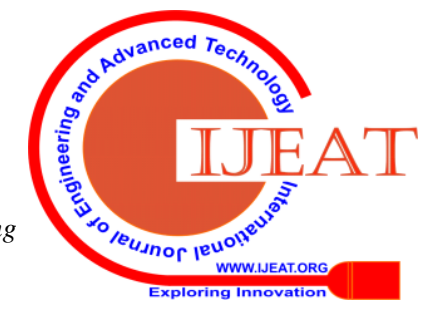




\section{CONCLUSION}

This paper has sought to present a comprehensive control solution effective for the coordinated operation of multiple microgrids as a network. The coordination is realized in both the grid and islanded modes of microgrid operation using droop characteristics.

Whenever there is a microgrid having demand more than its local generation, the neighboring microgrids in the network participate in active power sharing to avoid load shedding. The control solution also accounts for the stabilization of voltages and frequency that go through oscillations following a sudden load change. Moreover, to acquire the best system performances, the parameters of the controllers are optimized using a PSO algorithm that relies on a carefully defined objective function reflecting load sharing and power quality performance of the network. Thus, the proposed controllers drive the networked system to work healthy over a wide range of its operating modes. The simulation results confirm the capability of the proposed control strategies in enhancing the reliability and power quality of a weak power system that a networked microgrid becomes in its islanded mode of operation. The use of the proposed 3-level VSIs instead of the conventional 2-level VSIs in this work has led to achieve better output waveforms with reduced harmonic distortions.

\section{REFERENCES}

1. IRENA, "Renewable capacity statistics 2018," International Renewable Energy Agency (IRENA), Tech. Rep., Abu Dhabi, Mar. 2018, [Online].

2. Z. Li, M. Shahidehpour, F. Aminifar, A. Alabdulwahab, and Y. Al-Turki, "Networked microgrids for enhancing the power system resilience," Proceedings of the IEEE, vol. 105, no. 7, pp. 1289-1310, Jul. 2017.

3. S. Backhaus, L. Dobriansky, S. Glover, and et al., "Networked microgrids scoping study," Los Alamos National Laboratory, Tech. Rep., Jan. 2016.

4. Y. Li, P. Zhang, and P. B. Luh, "Formal analysis of networked microgrids dynamics," IEEE Trans. Power Syst., vol. 33, no. 3, pp. 3418-3427, May 2018.

5. H. M. A. Antunes, S. M. Silva, D.I. Brandao, R.V. Ferreira, A. A. P. Machado, and B. J. C. Filho,"A new multifunctional converter based on a series compensator applied to AC microgrids,"Int J Electr Power Energy Syst, 102;160-70, 2018.

6. A. Bernstein, L. Reyes-Chamorro, J.-Y. Le Boudec, and M. Paolone, "A composable method for real-time control of active distribution networks with explicit power setpoints. Part I: Framework," Electric Power Systems Research, vol. 125, pp. 254-264, 2015.

7. S. Adhikari and F. Li, "Coordinated V-f and P-Q control of solar photovoltaic generators with MPPT and battery storage in microgrids," IEEE Trans. Smart Grid, vol. 5, no. 3, pp. 1270-1281, 2014.

8. J. M. Guerrero, M. Chandorkar, T. Lee, and P. C. Loh, "Advanced Control Architectures for Intelligent Microgrids; Part I: Decentralized and Hierarchical Control," IEEE Trans. Ind. Electron., vol. 60, no. 4, pp. 1254-1262, Apr. 2013.

9. M. S. Golsorkhi and D. D. C. Lu, "A Control Method for InverterBased Islanded Microgrids Based on V-I Droop Characteristics," IEEE Trans. Power Del., vol. 30, no. 3, pp. 1196-1204, Jun. 2015.

10. E. Dall'Anese and A. Simonetto, "Optimal Power Flow Pursuit," IEEE Trans. Smart Grid, vol. 9, no. 2, pp. 942-952, Mar. 2018.

11. K. Baker, A. Bernstein, E. Dall'Anese, and C. Zhao, "NetworkCognizant Voltage Droop Control for Distribution Grids," IEEE Transactions on Power Systems, vol. 33, no. 2, pp. 2098-2108, Mar. 2018.

12. S. D'Arco, J.A. Suul, and O.B. Fosso, "Small-signal modeling and parametric sensitivity of a virtual synchronous machine in islanded operation,"Int J Electr Power Energy Syst, 72:3-15, 2015.

13. S. Bacha, I. Munteanu, and A. I. Bratcu, Power Electronic Converters Modeling and Control, London, U.K.: Springer, 2014, vol. 5.

14. M. G. Villalva, T. G. de Siqueira, and E. R. Filho, "Voltage regulation

Published By:

Blue Eyes Intelligence Engineering

\& Sciences Publication

(C) Copyright: All rights reserved. pp. 56-64, Dec. 2018. of photovoltaic arrays: Small-signal analysis and con
Power Electron., vol. 3, no. 6, pp. 869-880, 2010.

15. K. Przystupa, "Tuning of PID controllers- approximate methods," Advances in Science and Technology Research Journal, vol. 12, no. 4,

16. N. Amin, A. Q. Marowan, and B. C. Ghosh, "Application of Genetic Algorithm in Power System Optimization with Multi-type FACTS," International Journal of Scientific and Research Publications (IJSRP), vol. 7, no. 5, pp. 748-754, May 2017.

17. Z. Wang, B. Chen, J. Wang, and C. Chen, "Networked microgrids for self-healing power systems," IEEE Trans. Smart Grid, vol. 7, no. 1, pp. 310-319, Jan. 2016.

18. M. Shahidehpour, Z. Li, S. Bahramirad, Z. Li, and W. Tian, "Networked microgrids: exploring the possibilities of the IIT-Bronzeville grid," IEEE Power and Energy Magazine, vol. 15, no. 4, pp. 63-71, Jul. 2017.

19. M. Ross, C. Abbey, F. Bouffard, and G. Jos, "Multiobjective optimization dispatch for microgrids with a high penetration of renewable generation," IEEE Trans. Sustainable Energy, vol. 6, no. 4, pp. 1306- 1314, Oct. 2015

20. G. Liu, M. R. Starke, B. Ollis, and Y. Xue, "Networked microgrids scoping study," Oak Ridge National Laboratory, Tech. Rep., Oct. 2016.

21. K. V. Krishna, H. M. Suryawanshi, A. B. Shitole, and T. Ajmal, "Comparison between 2-level and 3-level grid connected inverters implemented using SRF PLL technique," in 2015 International Conference on Energy, Power and Environment: Towards Sustainable Growth (ICEPE), India, 2015, pp. 345-350.

22. S. Bourbour, F. Shahnia, and A. Ghosh, "Selection of a suitable microgrid to couple with an overloaded neighboring microgrid based on decision making," in 47th North American Power Symposium (NAPS), Charlotte, USA, 2015, pp.1-6.

23. Z. Wang, B. Chen, J. Wang, M. M. Begovic, and C. Chen, "Coordinated energy management of networked microgrids in distribution systems," IEEE Trans. Smart Grid, vol. 6, no. 1, pp. 45-53, Jan. 2015.

24. R. Eberhart and J. Kennedy, "A new optimizer using particle swarm theory," in Proc. 6th Int. Symp. Micro Mach. Hum. Sci. (MHS), Japan, 1995, pp. 39- 43.

25. B. Borowska, "Dynamic Inertia Weight in Particle Swarm Optimization," Advances in Intelligent Systems and Computing II, vol. 689, pp. 79-88, Springer, Cham, 2018.

26. H. Alhelou, M.-E. Hamedani-Golshan, R. Zamani, E. Heydarian-Forushani, and P. Siano, "Challenges and opportunities of load frequency control in conventional, modern and future smart power systems: a comprehensive review," Energies, vol. 11, no. 10, p. 2497, Sep. 2018. 